\title{
Feature
}

\section{Endovascular Options in the Management of Complex Aortic Problems}

\author{
OSVALDO J. YANO, M.D., ${ }^{1}$ MICHAEL L. MARIN, M.D., ${ }^{1}$ \\ and LARRY HOLLIER, M.D.'
}

ABSTRACT The eligibility for endovascular stent-graft repair in patients with aortic pathologies is dictated by the presence of favorable vascular anatomy. The two main anatomic features predicting successful repair are the presence of adequate attachment sites for the device anchoring and the availability of the relatively normal access vessels for safe device navigation. This chapter describes some of the complex vascular anatomies, which can initially impede aortic endovascular repair, and the maneuvers to circumvent these initial obstacles, increasing the overall pool of patients eligible for endovascular repair of complex aortic diseases.

Keywords Abdominal aortic aneurysm, endoluminal graft, stent graft

In the United States, there are approximately 200,000 new cases of abdominal aortic aneurysms (AAAs) diagnosed per year, and approximately 50,000 patients per year who have operations to have their aneurysm repaired. Thoracic aortic aneurysms have been diagnosed more frequently than in past, perhaps due to more liberal usage of imaging tests.

With aging of the population, the prevalence of aortic aneurysms is increasing. One in ten men over the age of 80 has enlargement of the abdominal aorta. ${ }^{1}$ The natural course of the aneurysms is to enlarge and rupture as the aneurysmal transverse diameter grows. Beginning with the origi-

Perspectives in Vascular Surgery and Endovascular Therapy, Volume 14, Number 1, 2001. Address for correspondence and reprint requests: Larry Hollier, M.D., Department of Surgery, Mount Sinai Medical Center, One Gustave

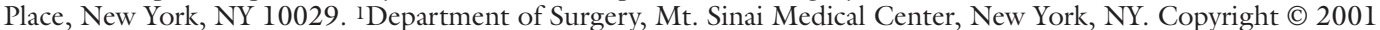
by Thieme Medical Publishers, Inc., 333 Seventh Avenue, New York, NY 10001, USA. Tel: +1(212) 584-4662. 1531-0035,p;2001,14,01,001,012,ftx,en;pvs00104x. 
nal report in 1951 by Dubost and collaborators, open surgical repair of AAA has remained the gold standard for more than 40 years. ${ }^{2}$ However, increasingly patients with aortic aneurysms are generally elderly and often have associated comorbidities, which categorizes them as a high risk for openconventional surgery. Although open surgery can be performed with a $5 \%$ mortality rate in many population studies, patients experience considerable morbidity up to $30 \%$ and slow recovery. ${ }^{3}$ Therefore, many patients and vascular surgeons have great enthusiasm in applying minimally invasive techniques, such as endovascular stent grafting, to repair aortic aneurysms, especially when the thoracic aorta is involved in the population at high risk for surgery.

One may speculate that the introduction of endoluminal grafts to treat aortic aneurysms has augmented the number of eligible patients suitable for repair because of the procedure's low invasiveness. However, this remains to be proven true.

Several published studies have demonstrated promising early therapeutic success, which is defined as total exclusion of the aneurysm from the arterial circulation with regression of the aneurismal sac diameter. Despite the initial optimism, durability of repair and stent-graft longevity has remained the principal unresolved matter and should be taken into account when choosing endovascular stent grafting as the treatment option.

\section{PATIENT ELIGIBILITY}

There are several steps involved in determining whether a patient is eligible to undergo stent-graft repair of an aortic aneurysm. These criteria are based solely on the anatomic configuration of the aorta and its branches. ${ }^{4}$ For the sake of simplicity, these main anatomic determinants can be divided into two categories:

1. Arteries suitable for device navigation

2. Adequate arterial implantation sites

\section{Infrarenal Aorta}

Although aortic aneurysms have a predilection to involve the infrarenal segment, in the majority of cases the juxtarenal segment is spared dilatation. A suitable diameter and length of proximal aortic neck is encountered in the majority of patients. Disease-free aorta must be used to ensure secure proximal attachment and long-term durability of the repair. There are, however, several anatomic characteristics that make management of the juxtarenal region challenging. The angiographic definition of an adequate proximal neck is defined by the presence of a normal caliber nonangulated vessel. The neck ideally should have a length of 10 to $15 \mathrm{~mm}$. Modifications of device design, however, by using proximal open stent design for transrenal fixation, 
can allow secure deployment in some patients with a proximal aortic neck of $0.5 \mathrm{~cm}$.

\section{Thoracic Aorta}

Thoracic aortic aneurysms do not have the same anatomic predilection as abdominal aorta. This behavior makes device customization more difficult and almost every anatomy random. At this moment, the only aneurysms eligible to be treated by stent-graft exclusion are the ones limited to the descending aorta. These aneurysms should have sufficient length for device anchoring distal to the arch vessels as well as proximal from the visceral branches of the aorta.

\section{Iliac Arteries}

To deploy a device, one must be able to negotiate the delivery system through the iliac vessels. The currently used devices are carried through a delivery system with an outer diameter ranging from 18 to $28 \mathrm{Fr}$. The malleability of the delivery system that contains the stent graft varies from device to device, but the ultimate goal is to be able to reach the target vessel segment with a minimum of iliac artery injury. With aging, iliac vessel atherosclerosis is more pronounced due to stenosis, calcification, and tortuosity. A few studies in the literature have quoted an incidence of up to $47 \%$ of significant iliac pathology complicating device navigation to the target area in an otherwise straightforward anatomy for endovascular repair. ${ }^{5}$ In our experience over 8 years, 35 patients with significant iliac artery disease or $7 \%$ of a total of 390 patients with aortic aneurysms required supplemental surgical interventions on the iliac vessels to correct what was considered a major impediment in their eligibility as candidates for endovascular stent graft. ${ }^{4}$

\section{COMPLEX AORTIC ANEURYSMS}

This article focuses on the definition of difficult vascular anatomy, which initially may not be suitable for catheter-based therapy. One must be conscious of the fact that technology will rapidly overcome the various endovascular limitations that are currently present, thus allowing more patients to be treated by this minimally invasive technique. Meanwhile, this article also describes various situations in which secondary surgical techniques can be combined with endovascular techniques to create a hybrid type of intervention, both endovascular and surgical, with the intent of optimizing inadequate anatomy for endovascular repairs. To approach the various complex aortic anatomies amenable for endovascular stent grafting, there are three main issues: (1) to delineate the measures used to overcome femoral-iliac arterial disease as a significant impeding factor to adequate endograft inser- 
tion for the treatment of thoracic and abdominal aortic aneurysms; (2) to describe the various adjunctive maneuvers used to enhance endograft implantation sites by either creating or extending the stent-graft attachment zones and to describe the overall incidence of patients requiring these maneuvers; and (3) to provide a detailed description of the various auxiliary maneuvers needed in this series of patients to circumvent these initial obstacles and make these patients eligible for endovascular stent-graft therapy.

\section{Facilitating Endovascular Device Delivery}

Coexisting atherosclerosis in the iliofemoral system is the most common problem encountered in these patients, and this system is also the most common access site used for device insertion. When choosing an access site, the arteries must be relatively free of disease, thus allowing navigation of the delivery system through them to the aneurysmal aorta. However, currently available devices have large and nonsteerable delivery systems, which can cause iliofemoral injuries during insertion of the device at a rate of $5 \%$ to $17 \% .^{6-8}$ Note that the majority of these patients did not have significant iliofemoral disease identified prior to the endovascular intervention. Unsuitable iliofemoral conduits are particularly problematic with large devices, which are required for thoracic aortic aneurysms. In some instances, despite the presence of a relative disease-free femoroiliac segment, these vessels are small to accommodate a large delivery system. This is especially common in female patients with thoracic aneurysms. In general, as a significant number of elderly patients with advanced atherosclerotic disease are treated by this therapeutic modality, access and navigational problems will become even more apparent as one of the main initial impediments to patient eligibility. The main factors prompting the use of auxiliary techniques were access vessel tortuosity or the presence of occlusive disease. The following maneuvers are described according to the various arterial diseases and their correspondent intervention used to correct the lesion:

1. Iliac artery angioplasty. Although rare, patients with short segment stenotic iliac lesions can be treated by balloon angioplasty of the iliac vessels prior to stent-graft insertion. Hemodynamically significant short lesions can be usually dealt with by careful passage of the device cone tip, which can gently dilate the plaque with minimal disruption of the overall vessel. In arteries requiring angioplasty, a 10- to $12-\mathrm{mm}$ Wallstent (Wallstent, Boston Scientific Corp., Natick, MA) can be deployed in the predilated segment to prevent arterial recoil following endograft deployment.

2. Endoluminal balloon endarterectomy. In patients with extensive external iliac artery stenosis, an endoluminal balloon endarterectomy can be performed to alleviate the occlusive process. The technique utilizes multiple balloon inflations throughout the occlusive external iliac 
arterial segment leading to plaque fractures and disattachment from the adventitia with subsequent removal of atheromatous debris via the femoral arteriotomy site. Subsequent stent insertion is used to prevent arterial restenosis. In our experience, this maneuver should be avoided in the presence of concentric calcification of the iliac vessels due to a high risk of arterial rupture.

3. Endoluminal stent graft. In patients with long-segment external iliac lesions, which are not amenable to endoluminal endarterectomy, an endoluminal PTFE conduit can be used to allow safe device navigation avoiding the risk for arterial rupture (Fig. 1). A Palmaz stent (Cordis, Johnson and Johnson Interventional Systems, Warren, NJ) is sutured into a 6-mm PTFE conduit and backloaded into a 6-Fr device system.

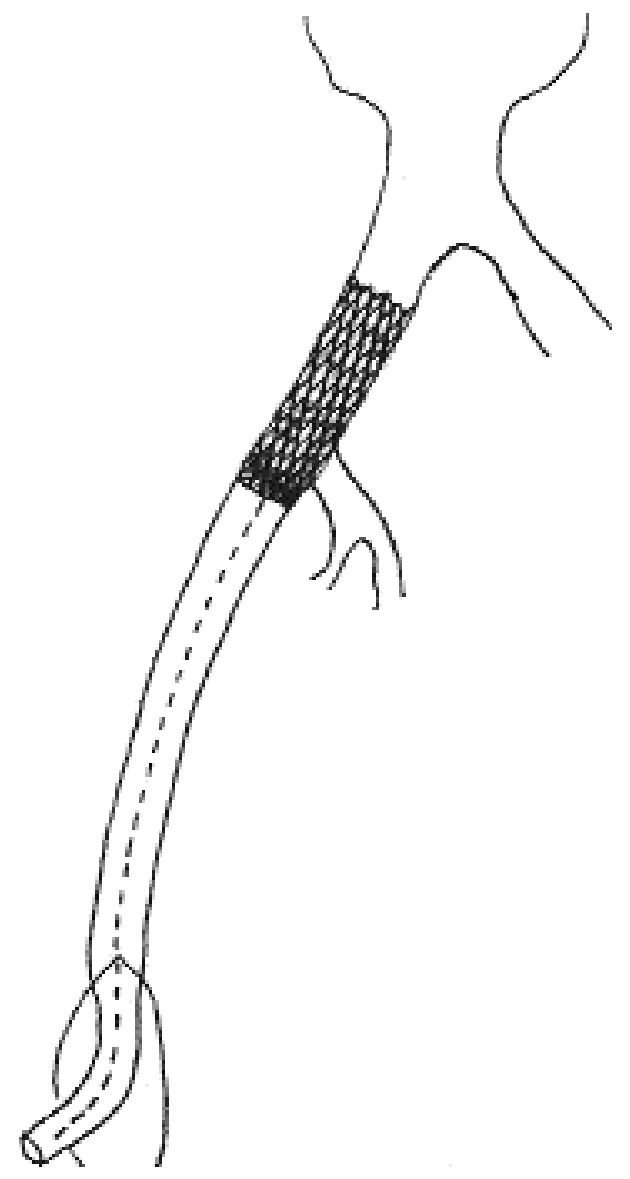

Fig. 1 An endoluminal iliofemoral conduit deployed in the iliac system with preservation of the internal iliac artery orifice. Multiple angioplasties can be performed safely from within the conduit, which is subsequently used as access for delivery of the aortic device. 
Under fluoroscopic guidance a Palmaz stent can be deployed over the orifice of the internal iliac artery with the graft extending to the external iliac artery. The entire length of the external iliac artery is then dilated from within the PTFE graft by using noncompliant balloon (6 to $8 \mathrm{~mm}$ in diameter) (Diamond, Boston Scientific, Natick, MA). The endoluminal conduit can then be implanted into the distal external iliac artery or hand-sewn anastomosis into the common femoral artery. This maneuver is very similar to the endoluminal endarterectomy; however, the multiple angioplasties can be performed in a safe manner from within the graft, minimizing the risk of leakage. Once this is completed, a stent graft can be navigated safely to its target segment within the aorta. Augmentation of distal extremity blood flow is also accomplished by this maneuver by terminating the graft in the common femoral artery.

4. External iliac artery straightening. In the majority of patients, the redundancy of the iliac artery is straightened by the use of stiff wires, which are routinely used to allow over-the-wire delivery of the device. However, in markedly tortuous external iliac arteries, extraredundant length has to be corrected prior to device insertion. Immediate recognition is imperative to avoid rupture of the iliac vessels during device navigation. The maneuver to correct this problem is simple; a digital retroperitoneal dissection can be performed with ligation of the circumflex branches of the external iliac artery. Once the artery is dissected free, the vessel can be pulled down to straighten the redundant arterial segment. Once mobilized, the redundant and tortuous segment can be excised, allowing safe device passage.

5. Iliofemoral bypass conduit. In patients with severe occlusive disease associated with marked tortuosity involving the entire length of the iliac artery, an iliofemoral bypass can be constructed through a retroperitoneal incision. This maneuver, although it requires a retroperitoneal dissection, is well tolerated by even frail patients and appears not to increase the overall morbidity of the procedure. The extraluminal iliofemoral bypass conduit serves to improve lower extremity arterial flow and to allow device navigation to the aorta through the bypass conduit. An iliofemoral bypass conduit can be also constructed in patients with small-caliber external iliac arteries (less than $5 \mathrm{~mm}$ in transverse diameter), which are considered small to accommodate a relatively large delivery system. In these patients, the bypass is removed following endovascular aneurysm exclusion by the stent graft. This situation is particularly common in women with thoracic aortic aneurysms.

\section{Implantation Site}

Endovascular treatment of arterial aneurysms requires normal arterial segments immediately adjacent to the aneurysmal area for adequate device 
anchoring. Inadequate proximal implantation site can be complicated by the presence of short-length attachment sites, which can cause insufficient apposition of stent graft to normal arterial wall, compromising the longevity of the repair. Wolf et al. have published the eligibility rate of patients with aortic aneurysms to have endovascular repair based on a large number of patients studied in the phase II AneuRx trial published in this journal. ${ }^{9}$ Inadequate proximal implantation site was the main cause for excluding patients for receiving endografting of AAA. However, in interpreting the data, one must consider the fact that only one stent graft was used in this cohort and patients were strictly controlled by rigid inclusion criteria of the trial.

It is well known that patients with abdominal or thoracic aortic aneurysms have other concomitant arterial pathologies, which may complicate or even exclude them from receiving endovascular stent-graft therapy. ${ }^{10-13}$ Distal implantation sites can be difficult due to the presence of common iliac artery aneurysms, which not only complicate selection of an adequate implantation site but also preservation of the internal iliac arteries and prograde pelvic flow. ${ }^{14}$ In section, we describe the device attachment site challenges and some of the maneuvers used to ameliorate these problems. To improve the overall condition of the attachment sites, modifications of the proximal and distal implantation sites can be performed. For the sake of simplicity, these procedures can be divided into two groups:

1. Direct manipulation to enhance the target site to achieve a complete seal of the aneurysm by the device

2. Bypass to essential aortic branches, thereby extending the inadequate neck to a proximal more favorable aortic segment

The specific anatomic presentations and their corresponding maneuvers are as follows:

1. Preservation of pelvic arterial blood flow in patients with bilateral iliac artery aneurysms: Almost $20 \%$ of patients with AAA have concomitant dilatation of the iliac arteries. This represents a potential restriction because it limits the choice for distal attachment sites. Concomitant existence of iliac artery aneurysms can be dealt by simply occluding the internal iliac artery ipsilateral to the aneurysms with subsequent deployment of the device into the external iliac artery. This procedure is usually well tolerated in the majority of patients, assuming that there is a normal deep femoral and contralateral internal iliac artery to perfuse the occluded internal iliac in a retrograde fashion. This problem is more complicated when both iliac arteries are aneurysmal. In this case, several maneuvers can be performed to preserve prograde pelvic circulation. Patients with bilateral common iliac artery aneurysm (Fig. 2) can have their internal iliac artery orifice relocated to a distal portion to allow stent-graft implantation in the external iliac artery segment. ${ }^{14}$ The second 


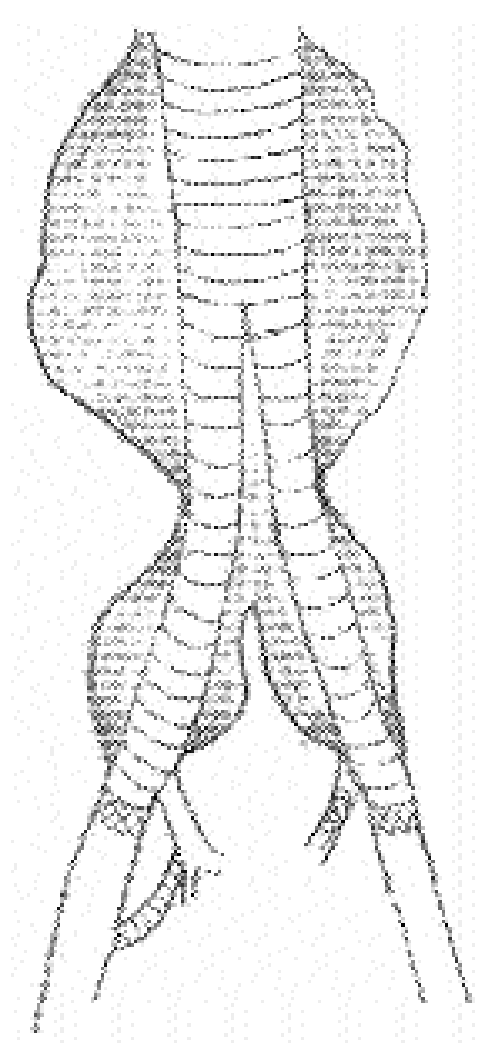

Fig. 2 In cases where bilateral iliac arteries are aneurysmal, relocation of the internal iliac artery can be performed to further distal segment, allowing deployment of the device in the external iliac artery while preserving prograde blood flow to the pelvis.

option would be to do plication of the iliac artery bifurcation to preserve one internal iliac to allow perfusion from the femorofemoral bypass in aorto-uni-iliac systems. Some advocate bilateral intentional occlusion of the internal iliac arteries; in our experience over $50 \%$ of patients have complains of chronic buttock claudication following this procedure. ${ }^{15}$

2. Subclavian artery transposition: Subclavian artery transposition can be used in patients who have their thoracic aneurysms beginning in close proximity to the subclavian artery (Fig. 3). This maneuver allows the endovascular stent graft to cover the original orifice of the artery to assure sufficient apposition of the stent graft to the proximal neck. Occlusive lesions of these vessels treated by constructing subclaviancarotid bypass carries an excellent long-term patency, as described by Berguer and colleagues. 16 Thus, one would expect comparable results in a similar patient cohort. Prior conformation of left common carotid artery free of significant occlusive disease must be done. This procedure is well tolerated due to its small incision in the neck.

3. Elephant trunk type aortic arch repair used as proximal implantation site for endograft: In our experience, three patients who had a previous elephant trunk repair for acute type A aortic dissections had 


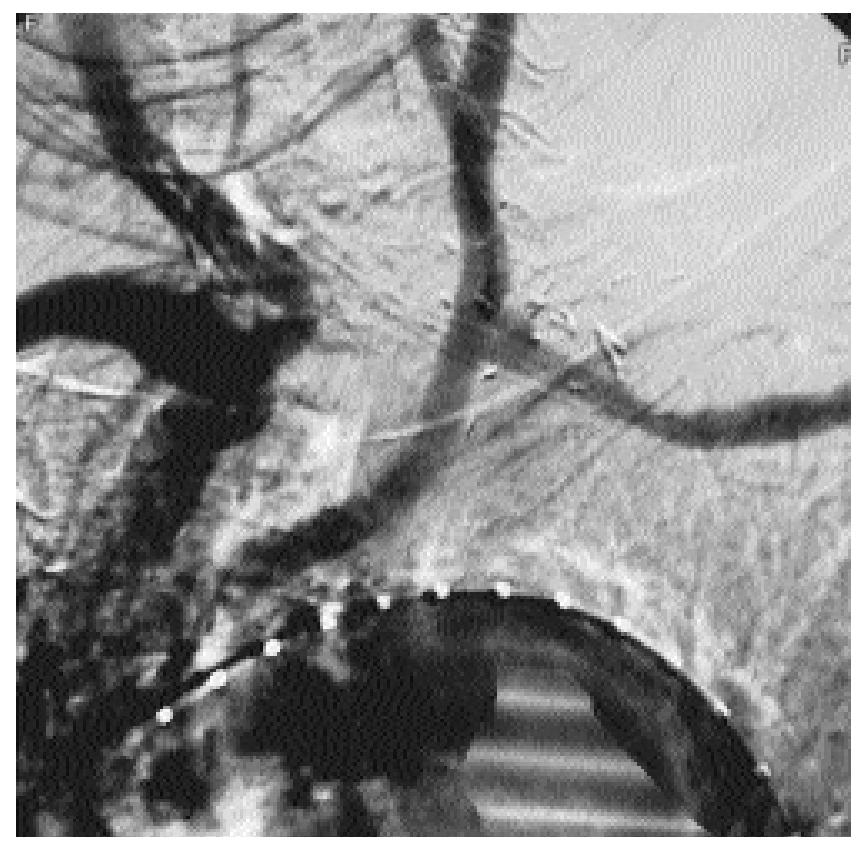

Fig. 3 For thoracic aortic aneurysms originating at the subclavian artery orifice, construction of a subclavian-carotid bypass has proved to be a useful maneuver in creating the necessary neck length to allow safe deployment of a device.

their descending aortic aneurysm repaired by stent-graft exclusion. Following the initial repair, these patients went on to develop large descending aortic aneurysms necessitating intervention. In these cases the graft material from the arch reconstruction was used as proximal implantation site or neck to anchor the stent graft (Fig. 4). Likewise, the lack of long-term results on graft diametric changes is the main concern following this type of procedure.

4. Staged extraanatomic visceral vessel reconstruction and subsequent endovascular aortic grafting: In a patient with high surgical risk due to multiple reoperations for a type IV thoracoabdominal aneurysm, construction of an extraanatomic bypass to the superior mesenteric artery from supraceliac aorta, in conjunction with bilateral renal arteries bypass from the iliac arteries using 8 -mm diameter graft, was performed prior to the aortic stent-graft repair. Following a brief period of recovery, the thoracoabdominal aneurysm stent-graft repair was performed with inconsequential coverage of otherwise essential aortic branches (Fig. 5).

\section{FUTURE APPROACHES}

New generation devices should have the ability to configure in a perfect plane to the aneurysm neck even in a presence of marked angulations, which will 

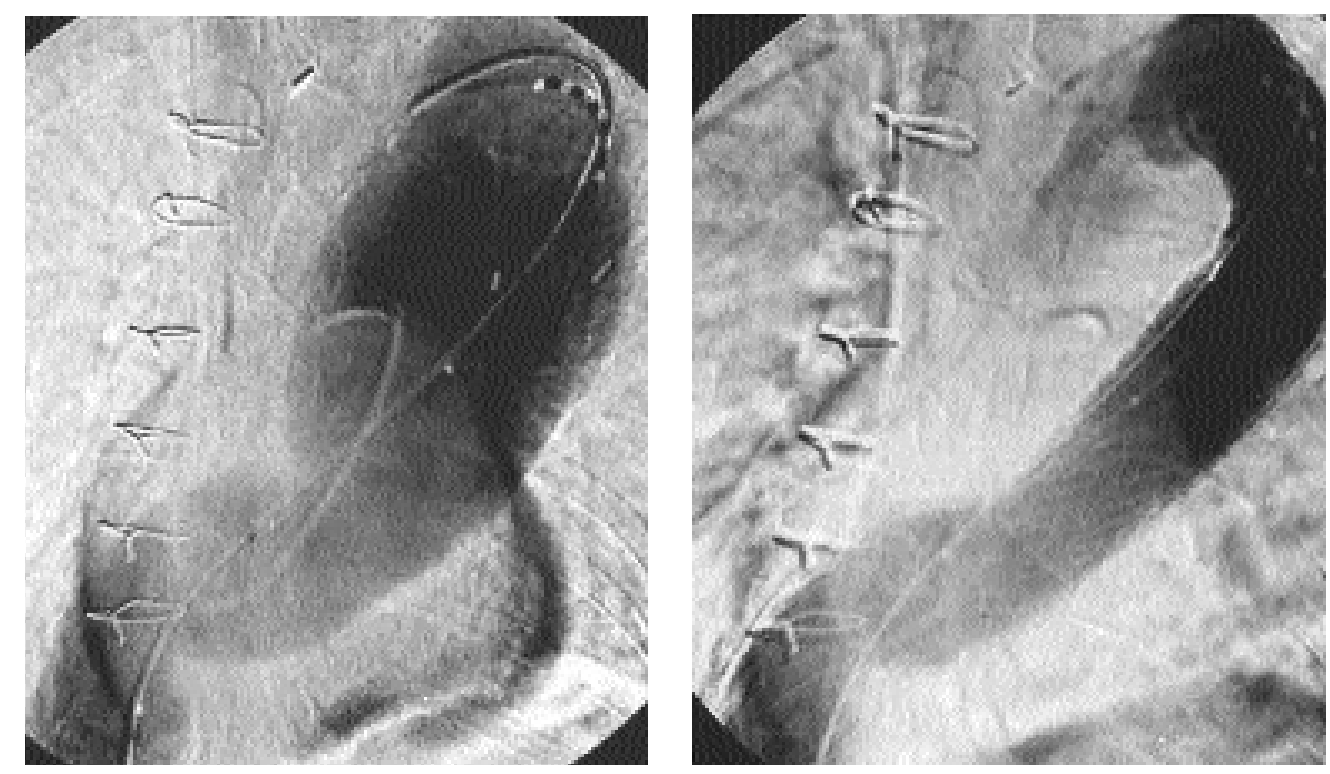

Fig. 4 The second stage of a thoracic aortic aneurysm following an elephant trunk type repair can be performed with a stent graft, as shown prior to and following device deployment.

allow optimal stent-graft positioning within the target vessel. Although in an experimental phase, new generation devices are being developed with the capability to treat and preserve aortic side branches, overcoming one of the limitations of currently used endovascular devices. Australian investigators Lawrence-Brown et al. ${ }^{17}$ have extensive experience in customizing branched stent grafts for suprarenal aortic aneurysms with successful exclusion of the aneurysm sac from the arterial circulation. This technique is called fenestration of stent grafts, in which the target aortic branch is aligned with a fenestration created on a main graft. Through the fenestrated role a second device is deployed into the branch vessel to connect the vessel with the main device, achieving tight seal without sacrificing blood flow from an essential aortic branch. Great technical skills are required to become proficient in this type of procedure. Dr. Inoue in Japan has expanded the stent-graft interventional capability to treat aortic aneurysms and dissections. ${ }^{18}$ Although similar to the Australian work, aortic arch work involves retrograde access of the subclavian and carotid vessels for optimal anchoring of the branched device. Dr. Inoue and his group have published their initial experience in 15 patients with aortic arch dissections and aneurysms with good short-term success. ${ }^{18}$ As imaging capabilities allow us to exam diseased anatomic beds more precisely, we believe that many new generation stent grafts will have easier delivery systems capable of navigating through tortuous vessels with precise deployment, overcoming many of the presently existing limitations. However, the exis- 


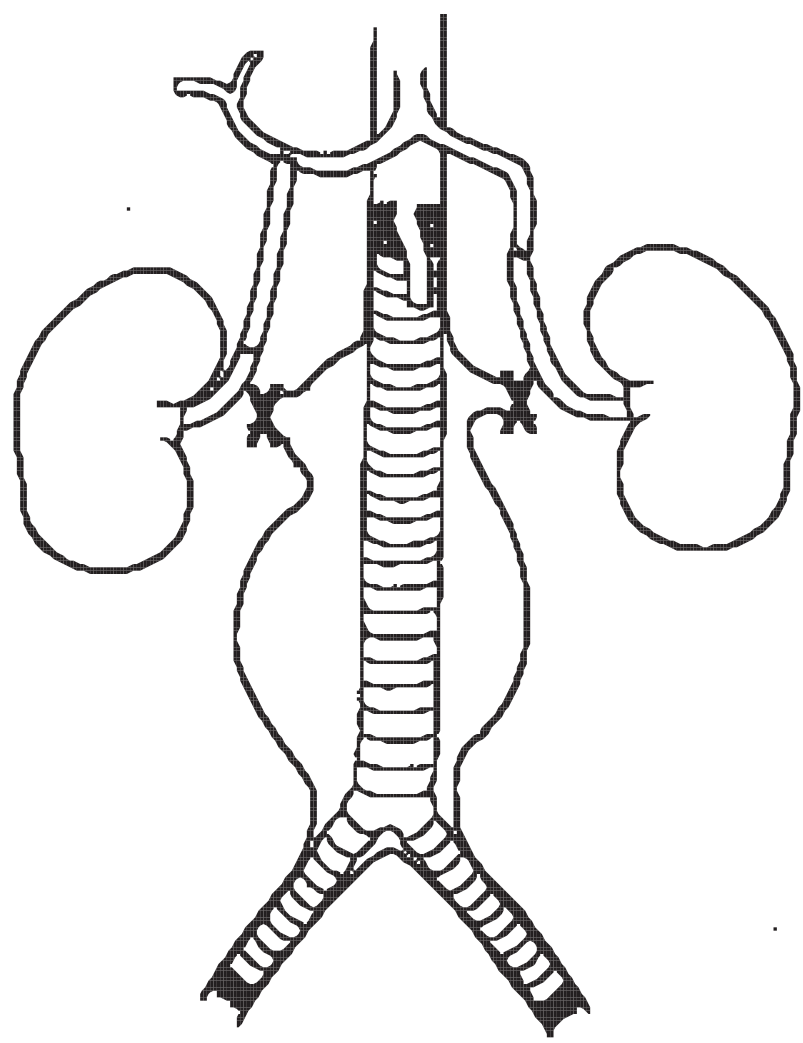

Fig. 5 Extraanatomic bypass to relocate renal artery inflow, expanding the proximal attachment site to a more favorable position.

tence of this technology will mainly depend on the long-term results regarding device longevity and the overall durability of the endovascular repair.

\section{REFERENCES}

1. Bengtsson H, Bergqvist D, Ekberg O, Janzon L. A population based screening of AAAs. J Vasc Surg 1991;5:53-57

2. Bengtsson H. Abdominal aortic aneurysm-aspects on epidemiology and screening. Ph.D. Thesis, Lund University, 1992

3. Johnston KW. Multicenter prospective study of nonruptured abdominal aortic aneurysm. Part II: variables predicting morbidity and mortality. J Vasc Surg 1989;9:437-447

4. Yano OJ, Morrisey N, Teodorescu V, et al. Supplemental surgical techniques to facilitate endovascular repair of abdominal and thoracic aortic aneurysms. J Vasc Surg, in press

5. Henretta JP, Karch LA, Hodgson KJ, et al. Special iliac artery considerations during aneurysm endografting. Am J Surg 1999;178:212-218

6. Blum U, Voshage G, Lammer J, et al. Endoluminal stent-grafts for infrarenal abdominal aortic aneurysms. NEJM 1997;336:13-20

7. Mialhe C, Amicabile C, Becquemin JP. Endovascular treatment of infrarenal abdominal aneurysms by the Stentor system: preliminary results of 79 cases. Stentor Retrospective Study Group. J Vasc Surg 1997;26:199-209 
8. Stelter W, Umscheid T, Ziegler P. Three-year experience with modular stent-graft devices for endovascular AAA treatment. J Endovasc Surg 1997;4(4):362-369

9. Wolf GY, Fogarty TJ, Olcott C, et al. Endovascular repair of abdominal aortic aneurysms: eligibility rate and impact on the rate of open repair. J Vasc Surg 2000;32:519-523

10. Armon MP, Yusuf SW, Latief K, et al. Anatomical suitability of abdominal aortic aneurysms for endovascular repair. Br J Surg 1997;84(2):178-180

11. Chuter TA, Reilly LM. Surgical reconstruction of iliac arteries prior to endovascular aortic aneurysm repair. J Endovasc Surg 1997;4:307-311

12. Naslund TC, Edwards WH, Neuzil DF, et al. Technical complications of endovascular abdominal aortic aneurysm repair. J Vasc Surg 1997;26:502-509

13. Chuter TA, Reilly LM, Kerlan RK, et al. Endovascular repair of abdominal aortic aneurysms: getting out of trouble. Cardiovasc Surg 1998;6:232-239

14. Parodi JC, Ferreira M. Relocation of the iliac artery bifurcation to facilitate endoluminal treatment of abdominal aortic aneurysms. J Endovasc Surg 1999;6:342-347

15. Yano OJ, Morrisey N, Eisen L, et al. Intentional internal iliac artery occlusion to facilitate endovascular repair of aortoiliac aneurysms. J Vasc Surg 2000, in press

16. Berguer R, Kline RA. Methods for reconstruction of proximal subclavian artery lesions: transposition and bypass. Semin Vasc Surg 1996;9(2):98-104

17. Browne TF, Hartley D, Purchas S, et al. A fenestrated covered supra-renal stent. Eur J Vasc Endovasc Surg 1999;18(5):445-449

18. Inoue $\mathrm{K}$, Hosokawa $\mathrm{H}$, Iwase $\mathrm{T}$, et al. Aortic arch reconstruction by transluminally placed endovascular branched stent graft. Circulation 1999;100:316-321 
It is a great pleasure for me to comment on the article by Drs. Yano, Marin, and Hollier.

I have known Drs. Marin and Hollier for many years and I am familiar with their many achievements.

The topic of patient selection for endovascular repair of aortic aneurysms deserves great attention. The authors should be commended for their caution and their enthusiasm for this novel development. I remember how one of the authors (M.L.M.) took seriously the selection of very high risk patients to be treated with endografts. The first patient we treated together, who incidentally was the first patient treated in the United States, could hardly lie on his back. Because of his comorbid conditions, the anesthesiologist was leaving the room at the same time we were entering it.

Aneurysms create vectors of forces inside them that represent a great challenge for the integrity of endografts. Facing a complex problem can generate two kinds of reactions: One can decide that the problem is unsolvable, or one can assume that failures should be analyzed and solutions for problems proposed. The authors of this article chose the latter.

The problem of short necks is addressed and solutions proposed. Suprarenal fixation seems to be the solution not only for short necks but also for the potential problem of neck dilatation. The adventitia of the visceral segment of the aorta is much stronger than the adjacent aorta. In addition, endoleaks are dramatically reduced by suprarenal fixation.

In addressing the topic of device selection, the authors emphasize the "one size fits all" principle we used in the past; it is still valid for cases such as emergencies, in which no time is available to define dimensions.

In discussing patient eligibility, the authors emphasize the uncertainly of long-term stent-graft durability; this is the main point readers should take from this article. Unless the medical center participates in a clinical trial, standard risk patients are still better served by conventional surgery.

Based on one of their previous papers, ${ }^{1}$ the authors discuss filling defects found at the level of the neck of the aneurysm. They confirm the inability of current imaging modalities to distinguish plaque from thrombus.

I want to comment about my experience with short angulated necks: To be very precise in deploying endografts, it is essential to obtain an angiogram to guide the release of the endograft in the most appropriate way. It is useful

Perspectives in Vascular Surgery and Endovascular Therapy, Volume 14, Number 1, 2001. Address for correspondence and reprint requests: Juan Carlos Parodi, M.D., Institute CV de Buenos Aires, Blanco Encalada 1543, Buenos Aires, Argentina. ${ }^{1}$ Department of Vascular Surgery, Institute CV de Buenos Aires, Argentina. Copyright (C) 2001 by Thieme Medical Publishers, Inc., 333 Seventh Avenue, New York, NY 10001, USA. Tel: +1(212) 584-4662. 1531$0035, \mathrm{p} ; 2001,14,01,013,014$, ftx, en;pvs00115x. 
to plan in advance the position of the x-ray tube, studying the angulations and take-off of the renal arteries in the spiral computed tomography (CT) scan. The position and angulation of the neck added to the position of the renal arteries ostium will dictate where to place the $\mathrm{x}$-ray tube. Parallax is another subject to consider; to gain accuracy, the neck should be positioned at the center of the screen.

An important issue is to analyze how perpendicular the proximal end of the endograft lies in relation to the actual axis of the neck. A device opened in an oblique position in relation to the central line axis of the neck will increase the possibility of occluding the renal artery or landing inside the aneurysmal sac, creating an endoleak. This is a critical situation surgeons must face when dealing with short and angulated necks.

The need to develop endografts with side branches is well taken and will represent a significant step forward for this technology.

When problems arise, it is good to go back to the initial thinking when we developed endografts to treat aneurysms ${ }^{2,3}$ : All we have to do is replace with efficacy the surgical suture. The feasibility and safety of the method have already been proven; durability is still the main issue. The application of existing and future technology will create a system comparable to the old surgical suture.

\section{REFERENCES}

1. Gitlitz DB, et al. Impact of a neck thrombus on endovascular stent-graft insertion. 27th Annual Meeting of the Clinical Association for Vascular Surgery, Orlando, FL, 1999

2. Parodi JC, Palmaz, JC, Barone HD. Transfemoral intraluminal graft implantation for abdominal aortic aneurysms. Ann Vasc Surg 1991;5:491-499

3. Parodi JC. Endoluminal treatment of arterial diseases using a stent-graft combination: reflections 20 years after the initial concept. J Endovasc Surg 1997;4:3-4 
The authors appreciate the kind comments by Dr. Parodi. As a friend, colleague, and teacher he is always gracious and informative.

Dr. Parodi raises several pertinent points that deserve added discussion. Additional comments could be made regarding patient selection, recommendations for "ideal" device construction, and the development of future technology of management of side branches.

Most vascular surgeons now accept the fact that endovascular stent grafts can play a valuable role in the management of selected patients with abdominal aortic aneurysms; this is especially true for those patients with significant pro-morbid risk factors. Additionally, it is now evident that individual patient anatomic variables most accurately delineate those patients who are likely to undergo a safe and effective endovascular aneurysm repair compared to those who are likely to have perioperative complications and/or long-term failure. Additional experience and disseminated education regarding these issues will allow better patient selection and a higher degree of success with endovascular repair of aneurysms.

As additional information and experience continues to accrue worldwide, we are in a better position to identify those aspects of stent graft construction likely to be associated with greater long-term success. It appears evident today that transrenal, or even transvisceral fixation is advantageous in preventing distal graft migration. Thus far, crossing of visceral vessels with a bare stent appears to be associated with minimal morbidity, while at the same time allowing excellent fixation to the most stable segment of the aorta.

The development of side-branch technology certainly appears to be the next major step in the evolution of endovascular stent grafts. Devices with single side-branch capabilities are already undergoing clinical trials and it is only a short time before multi-branch technology will also be available. In the near future we can expect to extend the range of aneurysm treatment not only to complex infrarenal aneurysms, but even to some thoracoabdominal aneurysms involving all of the visceral vessels and even some aneurysms involving vessels of the aortic arch. It is fascinating to note that in the 10 years since Dr. Parodi's pioneering efforts with endovascular stent grafting, tremendous strides have been made. One has to believe that this is only a fraction of the advances that will occur over the next decade.

Perspectives in Vascular Surgery and Endovascular Medicine, Volume 14, Number 1, 2001. Copyright (C) 2001 by Thieme Medical Publishers, Inc., 333 Seventh Avenue, New York, NY 10001, USA. Tel: +1(212) 584-4662. 15310035,p;2001,14,01,015,016,ftx,en;pvs00116x. 
